\title{
The maximal and minimal ranks of matrix expression with applications
}

\author{
Zhiping Xiong, Yingying Qin ${ }^{*}$ and Shifang Yuan
}

* Correspondence: qinyy04@163. com

Department of Mathematics, Wuyi University, Jiangmen 529020, P.R. China

\begin{abstract}
We give in this article the maximal and minimal ranks of the matrix expression $A$ $B_{1} V_{1} C_{1}-B_{2} V_{2} C_{2}-B_{3} V_{3} C_{3}-B_{4} V_{4} C_{4}$ with respect to $V_{1}, V_{2}, V_{3}$, and $V_{4}$. As applications, we derive the extremal ranks of the generalized Schur complement $A-B M^{(1)} C-D N^{(1)} G$ and the partial matrix $\left(A B M^{(1)} C D N^{(1)} G\right)$ with respect to the generalized inverse $M^{(1)} \varepsilon$ $M\{1\}$ and $N^{(1)} \in N\{1\}$.

AMS classifications: 15A03; 15A09; 15A24.
\end{abstract}

Keywords: matrix expression, maximal rank, minimal rank, generalized inverse, rank equality

\section{Introduction}

Let $C^{m \times n}$ be the set of all $m \times n$ complex matrices with complex entries. $I_{n}$ denotes the identity matrix of order $n$ and $O_{m \times n}$ denotes the $m \times n$ matrix of all zero entries (if no confusion occurs, we will omit the subscript). For a given a matrix $A \in C^{m \times n}$, the symbols $A^{*}$ and $r(A)$ will stand for the conjugate transpose and the rank of the matrix $A$, respectively. We recall that a generalized inverse $X \in C^{n \times m}$ of $A \in C^{m \times n}$ is a matrix which satisfies some of the following four Penrose equations [1]:
(1) $A X A=A$,
(2) $X A X=X$,
(3) $(A X)^{*}=A X$,
(4) $(X A)^{*}=X A$.

For a subset $\{i, j, \ldots, k\}$ of the set $\{1,2,3,4\}$, the set of $n \times m$ matrices satisfying the equations $(i),(j), \ldots,(k)$ from among the above four Penrose Equations (1)-(4) is denoted by $A\{i, j, \ldots, k\}$. A matrix $X$ from $A\{i, j, \ldots, k\}$ is called an $\{i, j, \ldots, k\}$-inverse of $A$ and is denoted by $A^{(i, j, \ldots, k)}$. In particular, an $n \times m$ matrix $X$ of the set $A\{1\}$ is called a g-inverse of $A$ and denoted by $A^{(1)}$. The unique $\{1,2,3,4\}$-inverse of $A$ is denoted by $A^{\dagger}$, which is called the Moore-Penrose inverse of $A$. Throughout this article, the abbreviated symbols $E_{A}$ and $F_{A}$ stand for the two projectors $E_{A}=I-A A^{\dagger}$ and $F_{A}=I-A^{\dagger} A$ of $A$, respectively. We refer the reader to [2,3] for basic results on the generalized inverses.

Given a matrix with some variant entries in it (often called partial matrix) or a matrix expression with some variant matrices in it, the rank of the partial matrix or matrix expression will vary with respect to the variant entries or variant matrices. Because the rank of matrix is an integer between 0 and the minimal of row and column numbers of the matrix, maximal and minimal ranks of partial matrix or matrix expressions must exist with respect to their variant entries or variant matrices. Many problems in matrix theory and applications are closely related to maximal and minimal

(C) 2012 Xiong et al; licensee Springer. This is an Open Access article distributed under the terms of the Creative Commons Attribution License (http://creativecommons.org/licenses/by/2.0), which permits unrestricted use, distribution, and reproduction in any medium, provided the original work is properly cited. 
possible ranks of matrix expressions with variant entries. For example, a matrix equation $A X B=C$ is consistent if and only if the minimal rank of $C-A X B$ with respect to $X$ is zero, see [4-6]; there is matrix $X$ such that the partial matrix $A X B$ of order $n$ is nonsingular if and only if the maximal rank of $A X B$ with respect to $X$ is $n$, see [7-11].

The maximal and minimal ranks of matrix expressions or partial matrix are two basic concepts in matrix theory for describing the dimension of the row or column vector space of matrix expressions or partial matrix, both of which are well understood and are easy to compute by the well-known elementary or congruent matrix operations, see $[5,7,8,10-16]$. These two quantities play an essential role in characterizing algebraic properties of matrices expressions or partial matrices. In fact, maximal and minimal ranks of matrix expressions or partial matrices have been the main objects of study in matrix theory and applications. Some previous systematical researches on maximal and minimal ranks of matrix expressions or partial matrices and their applications can be found in [17-20]. In recent years, the present author reconsidered the maximal and minimal ranks of matrix expressions or partial matrices by using some tricky operations on block matrices and generalized inverses of matrices, and obtained many new formulas for maximal and minimal ranks of matrix expressions or partial matrices and their applications, see [4,6,9,21-28].

In this article, given matrices $A \in C^{m \times n}, B_{i} \in C^{m \times p_{i}}, C_{i} \in C^{q_{i} \times n}, i=1,2,3,4$, we will present the maximal and minimal ranks of the matrix expression $A-B_{1} V_{1} C_{1}-B_{2} V_{2} C_{2}$ $B_{3} V_{3} C_{3}-B_{4} V_{4} C_{4}$ with respect to $V_{1}, V_{2}, V_{3}$, and $V_{4}$. As applications, the maximal and minimal ranks of the generalized Schur complement $A-B M^{(1)} C-D N^{(1)} G$ and the partial matrix $\left(A B M^{(1)} C D N^{(1)} G\right)$ with respect to the generalized inverse $M^{(1)} \in M\{1\}$ and $N^{(1)} \in N\{1\}$ are also considered. The results in this article extend the earlier studies by various authors, see, e.g., [4-6,11,16,18,21,25,26].

We first introduce some well-known results which will be used in this article.

Lemma $1.1[5,8,25]$. Let

$$
M=\left(\begin{array}{ccc}
A_{11} & A_{12} & X \\
A_{21} & A_{22} & A_{23} \\
Y & A_{32} & A_{33}
\end{array}\right)
$$

where $A_{i j} \in C^{m_{i} \times n_{j}}(1 \leq i, j \leq 3)$ are given, $X \in C^{m_{1} \times n_{3}}$ and $Y \in C^{m_{3} \times n_{1}}$ are two variant matrices. Then

$$
\begin{aligned}
& \max _{X, Y} r(M)=\min \left\{m_{3}+n_{3}+r\left(\begin{array}{ll}
A_{11} & A_{12} \\
A_{21} & A_{22}
\end{array}\right), m_{1}+n_{1}+r\left(\begin{array}{ll}
A_{22} & A_{23} \\
A_{32} & A_{33}
\end{array}\right),\right. \\
& \left.m_{1}+m_{3}+r\left(\begin{array}{lll}
A_{21} & A_{22} & A_{23}
\end{array}\right), n_{1}+n_{3}+r\left(\begin{array}{l}
A_{12} \\
A_{22} \\
A_{32}
\end{array}\right)\right\} \text {, } \\
& \min _{X, Y} r(M)=r\left(\begin{array}{ll}
A_{21} & A_{22} A_{23}
\end{array}\right)+r\left(\begin{array}{l}
A_{12} \\
A_{22} \\
A_{32}
\end{array}\right)+\max \left\{r\left(\begin{array}{ll}
A_{11} & A_{12} \\
A_{21} & A_{22}
\end{array}\right)-r\left(\begin{array}{l}
A_{12} \\
A_{22}
\end{array}\right)-r\left(A_{21} A_{22}\right),\right. \\
& \left.r\left(\begin{array}{ll}
A_{22} & A_{23} \\
A_{32} & A_{33}
\end{array}\right)-r\left(\begin{array}{l}
A_{22} \\
A_{32}
\end{array}\right)-r\left(A_{22} A_{23}\right)\right\} .
\end{aligned}
$$

Lemma 1.2 [2]. Let $A \in C^{m \times n}$. Then the expression of $\{1\}$-inverses of $A$ can be written as 


$$
A^{(1)}=A^{\dagger}+\left(I_{n}-A^{\dagger} A\right) W+Z\left(I_{m}-A A^{\dagger}\right),
$$

where $W \in C^{n \times m}$ and $Z \in C^{n \times m}$ are arbitrary.

Lemma 1.3 [9]. Let $A \in C^{m \times n}, B \in C^{m \times k}$, and $C \in C^{l \times n}$. Then

$$
\begin{aligned}
& \text { (1). } r(A B)=r(A)+r\left(E_{A} B\right)=r\left(E_{B} A\right)+r(B), \\
& \text { (2). } r\left(\begin{array}{l}
A \\
C
\end{array}\right)=r(A)+r\left(C F_{A}\right)=r\left(A F_{C}\right)+r(C)
\end{aligned}
$$

where $E_{A}=I_{m}-A A^{\dagger}$ and $F_{A}=I_{n}-A^{\dagger} A$.

\section{The maximal and minimal ranks of $A-B_{1} V_{1} C_{1}-B_{2} V_{2} C_{2}-B_{3} V_{3} C_{3}-B_{4} V_{4} C_{4}$}

In this section, we will present the maximal and minimal ranks of the linear matrix expression

$$
P\left(V_{1}, V_{2}, V_{3}, V_{4}\right)=A-B_{1} V_{1} C_{1}-B_{2} V_{2} C_{2}-B_{3} V_{3} C_{3}-B_{4} V_{4} C_{4},
$$

where $A \in C^{m \times n}, B_{i} \in C^{m \times p_{i}}, C_{i} \in C^{q_{i} \times n}, i=1,2,3,4$, are given matrices, with respect to four variant matrices $V_{i} \in C^{p_{i} \times q_{i}}, i=1,2,3,4$. Applying the formula (1) in Lemma 1.1 to the linear matrix expression in (4) and simplifying, we obtain the following result.

Theorem 2.1 Let $P\left(V_{1}, V_{2}, V_{3}, V_{4}\right)$ be given as (4). Then

$$
\max _{V_{1}, V_{2}, V_{3}, V_{4}} r\left(P\left(V_{1}, V_{2}, V_{3}, V_{4}\right)\right)=\min \left\{T_{1}, T_{2}, T_{3}, T_{4}\right\}
$$

where

$$
\begin{aligned}
r\left(P\left(V_{1}, V_{2}, V_{3}, V_{4}\right)\right)=r & \left(\begin{array}{ccccccccc}
O & O & O & O & O & O & O & I_{P_{4}} & -V_{4} \\
O & O & O & O & C_{4} & O & O & O & I_{q_{4}} \\
O & O & O & O & O & I_{P_{2}} & -V_{2} & O & O \\
O & O & O & O & C_{2} & O & I_{q_{2}} & O & O \\
O & B_{3} & O & B_{1} & A & B_{2} & O & B_{4} & O \\
O & O & I_{q_{1}} & O & C_{1} & O & O & O & O \\
O & O & -V_{1} & I_{P_{1}} & O & O & O & O & O \\
I_{q_{3}} & O & O & O & C_{3} & O & O & O & O \\
-V_{3} & I_{P_{3}} & O & O & O & O & O & O & O
\end{array}\right)-\sum_{i=1}^{4} p_{i}-\sum_{i=1}^{4} q_{i} \\
& r(T)-\sum_{i=1} p i-\sum_{i=1} q i,
\end{aligned}
$$

Proof. It is easy to verify by block Gaussian elimination that the rank of $P\left(V_{1}, V_{2}, V_{3}\right.$, $\left.V_{4}\right)$ in (4) can be expressed as

$$
\begin{aligned}
r\left(P\left(V_{1}, V_{2}, V_{3}, V_{4}\right)\right)=r & \left(\begin{array}{ccccccccc}
O & O & O & O & O & O & O & I_{P 4} & -V_{4} \\
O & O & O & O & C_{4} & O & O & O & I_{q 4} \\
O & O & O & O & O & I_{P 2} & -V_{2} & O & O \\
O & O & O & O & C_{2} & O & I_{q 2} & O & O \\
O & B_{3} & O & B_{1} & A & B_{2} & O & B_{4} & O \\
O & O & I_{q 1} & O & C_{1} & O & O & O & O \\
O & O & -V_{1} & I_{P 1} & O & O & O & O & O \\
I_{q 3} & O & O & O & C_{3} & O & O & O & O \\
-V_{3} & I_{P 3} & O & O & O & O & O & O & O
\end{array}\right)-\sum_{i=1}^{4} p i-\sum_{i=1}^{4} q i, \\
& r(T)-\sum_{i=1}^{4} p i-\sum_{i=1} q i
\end{aligned}
$$


where

$$
A \in C^{m \times n}, B_{i} \in C^{m \times p_{i}}, C_{i} \in C^{q_{i} \times n}, V_{i} \in C^{p_{i} \times q_{i}}, i=1,2,3,4
$$

$I_{p_{i}}, I_{q_{i}}, i=1,2,3,4$, are denotes the identity matrix of order $p_{i}$ and $q_{i}$, respectively.

$$
T=\left(\begin{array}{ccc}
O & E_{2} & -V_{4} \\
E_{1} & S & E_{3} \\
-V_{3} & E_{4} & O
\end{array}\right), S=\left(\begin{array}{ccccccc}
O & O & O & C_{4} & O & O & O \\
O & O & O & O & I_{p_{2}} & -V_{2} & O \\
O & O & O & C_{2} & O & I_{q_{2}} & O \\
B_{3} & O & B_{1} & A & B_{2} & O & B_{4} \\
O & I_{q_{1}} & O & C_{1} & O & O & O \\
O & -V_{1} & I_{p_{1}} & O & O & O & O \\
O & O & O & C_{3} & O & O & O
\end{array}\right)
$$

and

$$
\begin{array}{ll}
E_{1}=\left(\mathrm{OOOOOOI}_{q_{3}}\right)^{*}, & E_{2}=\left(\mathrm{OOOOOOI}_{p_{4}}\right), \\
E_{3}=\left(I_{q_{4}} \mathrm{OOOOOO}\right)^{*}, & E_{4}=\left(I_{p_{3}} \text { OOOOOO }\right) .
\end{array}
$$

According to this result, we have

$$
\max _{V_{1}, V_{2}, V_{3}, V_{4}} r\left(P\left(V_{1}, V_{2}, V_{3}, V_{4}\right)\right)=\max _{V_{1}, V_{2}, V_{3}, V_{4}} r(T)-\sum_{i=1}^{4} p_{i}-\sum_{i=1}^{4} q_{i} .
$$

Then applying the formula (1) in Lemma 1.1 to matrix $T$, we have

$$
\begin{gathered}
\max _{V_{3}, V_{4}} r(T)=\min \left\{p_{3}+q_{4}+r\left(\begin{array}{cc}
O & E_{2} \\
E_{1} & S
\end{array}\right) p_{4}+q_{3}+r\left(\begin{array}{cc}
S & E_{3} \\
E_{4} & O
\end{array}\right),\right. \\
\left.p_{4}+p_{3}+r\left(E_{1} S E_{2}\right), q_{3}+q_{4}+r\left(\begin{array}{c}
E_{2} \\
S \\
E_{4}
\end{array}\right)\right\} \\
=\min \left\{p_{3}+q_{4}+p_{4}+q_{3}+r\left(S_{1}\right), p_{4}+q_{3}+q_{4}+p_{3}+r\left(S_{2}\right),\right. \\
\left.p_{4}+p_{3}+q_{4}+q_{3}+r\left(S_{3}\right), q_{3}+q_{4}+p_{4}+p_{3}+r\left(S_{4}\right)\right\},
\end{gathered}
$$

where

$$
\begin{aligned}
& S_{1}=\left(\begin{array}{cccccc}
O & O & O & O & I_{P_{2}} & -V_{2} \\
O & O & O & C_{4} & O & O \\
O & O & O & C_{2} & O & I_{q_{2}} \\
O & B_{3} & B_{1} & A & B_{2} & O \\
I_{q_{1}} & O & O & C_{1} & O & O \\
-V_{1} & O & I_{p_{1}} & O & O & O
\end{array}\right), S_{2}=\left(\begin{array}{cccccc}
O & O & O & I_{P_{2}} & O & -V_{2} \\
O & O & C_{2} & O & O & I_{q_{2}} \\
O & B_{1} & A & B_{2} & B_{4} & O \\
I_{q_{1}} & O & C_{1} & O & O & O \\
O & O & C_{3} & O & O & O \\
-V_{1} & I_{p_{1}} & O & O & O & O
\end{array}\right) \\
& S_{3}\left(\begin{array}{ccccccc}
O & O & O & O & I_{P_{2}} & O & -V_{2} \\
O & O & O & C_{2} & O & O & I_{q_{2}} \\
O & B_{3} & B_{1} & A & B_{2} & B_{4} & O \\
I_{q_{1}} & O & O & C_{1} & O & O & O \\
-V_{1} & O & I_{p_{1}} & O & O & O & O
\end{array}\right), S_{4}=\left(\begin{array}{ccccc}
O & O & O & I_{P_{2}} & -V_{2} \\
O & O & C_{4} & O & O \\
O & O & C_{2} & O & I_{q_{2}} \\
O & B_{1} & A & B_{2} & O \\
I_{q_{1}} & O & C_{1} & O & O \\
O & O & C_{3} & O & O \\
-V_{1} & I_{p_{1}} & O & O & O
\end{array}\right)
\end{aligned}
$$

Again applying the formula (1) in Lemma 1.1, we get

$$
\begin{aligned}
\max _{V_{1}, V_{2}, V_{3}, V_{4}} r(T)= & \min \left\{p_{3}+q_{4}+p_{4}+q_{3}+\max _{V_{1}, V_{2}} r\left(S_{1}\right), p_{4}+q_{3}+q_{4}+p_{3}+\max _{V_{1}, V_{2}} r\left(S_{2}\right),\right. \\
& \left.p_{4}+p_{3}+q_{4}+q_{3}+\max _{V_{1}, V_{2}} r\left(S_{3}\right), q_{3}+q_{4}+p_{4}+p_{3}+\max _{V_{1}, V_{2}} r(S 4)\right\}
\end{aligned}
$$


and

$$
\begin{aligned}
& \max _{V_{1}, V_{2}} r\left(S_{1}\right)=\min \left\{p_{1}+q_{2}+q_{1}+p_{2}+r\left(\begin{array}{ccc}
O & O & C_{4} \\
O & O & C_{2} \\
B_{3} & B_{1} & A
\end{array}\right), p_{1}+q_{2}+q_{1}+p_{2}+r\left(\begin{array}{ccc}
O & C_{4} & O \\
B_{3} & A & B_{2} \\
O & C_{1} & O
\end{array}\right),\right. \\
& \left.p_{1}+q_{2}+q_{1}+p_{2}+r\left(\begin{array}{cccc}
O & O & C_{4} & O \\
B_{3} & B_{1} & A & B_{2}
\end{array}\right), p_{1}+q_{2}+q_{1}+p_{2}+r\left(\begin{array}{cc}
O & C_{4} \\
O & C_{2} \\
B_{3} & A \\
O & C_{1}
\end{array}\right)\right\} \\
& \max _{V_{1}, V_{2}} r\left(S_{2}\right)=\min \left\{p_{1}+q_{2}+q_{1}+p_{2}+r\left(\begin{array}{ccc}
O & C_{2} & O \\
B_{1} & A & B_{4} \\
O & C_{3} & O
\end{array}\right), p_{1}+q_{2}+q_{1}+p_{2}+r\left(\begin{array}{ccc}
A & B_{2} & B_{4} \\
C_{1} & O & O \\
C_{3} & O & O
\end{array}\right),\right. \\
& \left.p_{1}+q_{2}+q_{1}+p_{2}+r\left(\begin{array}{cccc}
B_{1} & A & B_{2} & B_{4} \\
O & C_{3} & O & O
\end{array}\right), p_{1}+q_{2}+q_{1}+p_{2}+r\left(\begin{array}{cc}
C_{2} & O \\
A & B_{4} \\
C_{1} & O \\
C_{3} & O
\end{array}\right)\right\}, \\
& \max _{V_{1}, V_{2}} r\left(S_{3}\right)=\min \left\{p_{1}+q_{2}+q_{1}+p_{2}+r\left(\begin{array}{cccc}
O & O & C_{2} & O \\
B_{3} & B_{1} & A & B_{4}
\end{array}\right)\right. \\
& p_{1}+q_{2}+q_{1}+p_{2}+r\left(\begin{array}{cccc}
B_{3} & A & B_{2} & B_{4} \\
O & C_{1} & O & O
\end{array}\right) \text {, } \\
& p_{1}+q_{2}+q_{1}+p_{2}+r\left(B_{3} B_{1} A B_{2} B_{4}\right) \text {, } \\
& p_{1}+q_{2}+q_{1}+p_{2}+r\left(\begin{array}{ccc}
O & C_{2} & O \\
B_{3} & A & B_{4} \\
O & C_{1} & O
\end{array}\right) \text {, } \\
& \max _{V_{1}, V_{2}} r\left(S_{4}\right)=\min \left\{p_{1}+q_{2}+q_{1}+p_{2}+r\left(\begin{array}{cc}
O & C_{4} \\
O & C_{2} \\
B_{1} & A \\
O & C_{3}
\end{array}\right), p_{1}+q_{2}+q_{1}+p_{2}+r\left(\begin{array}{cc}
C_{4} & O \\
A & B_{2} \\
C_{1} & O \\
C_{3} & O
\end{array}\right),\right. \\
& p_{1}+q_{2}+q_{1}+p_{2}+r\left(\begin{array}{ccc}
O & C_{4} & O \\
B_{1} & A & B_{2} \\
O & C_{3} & O
\end{array}\right), p_{1}+q_{2}+q_{1}+p_{2}+r\left(\begin{array}{c}
C_{4} \\
C_{2} \\
A \\
C_{1} \\
C_{3}
\end{array}\right) \text {. }
\end{aligned}
$$

Substituting (8)-(11) into (7) and (6) yield (5).

Recall a simple fact that a matrix equation $A X B=C$ is consistent for every variant matrices $X$, if and only if the maximal rank of $C$ - $A X B$ with respect to $X$ is zero. Thus, by Theorem 2.1 we can immediately obtain the following result.

Corollary 2.2 Let $P\left(V_{1}, V_{2}, V_{3}, V_{4}\right)$ be given as (4). Then the matrix equation $A=$ $B_{1} V_{1} C_{1}+B_{2} V_{2} C_{2}+B_{3} V_{3} C_{3}+B_{4} V_{4} C_{4}$ holds for any $V_{1}, V_{2}, V_{3}$, and $V_{4}$ if and only if $T_{1}=O$ or $T_{2}=O$ or $T_{3}=O$ or $T_{4}=O$.

Because the right side of (5) are just composed by ranks of block matrices, they can be easily simplified by block Gaussian elimination when the given matrices in (4) satisfy some restrictions.

Theorem 2.3 Let $P\left(V_{1}, V_{2}, V_{3}, V_{4}\right)$ be given as (4) and let $R\left(B_{1}\right) \subseteq R\left(B_{2}\right), R\left(B_{3}\right) \subseteq R$ $\left(B_{4}\right), R\left(B_{1}\right) \subseteq R\left(B_{2}\right), R\left(B_{3}\right) \subseteq R\left(B_{4}\right), R\left(C_{2}^{*}\right) \subseteq R\left(C_{1}^{*}\right), R\left(C_{4}^{*}\right) \subseteq R\left(C_{3}^{*}\right)$ Then

$$
\max _{V_{1}, V_{2}, V_{3}, V_{4}} r\left(P\left(V_{1}, V_{2}, V_{3}, V_{4}\right)\right)=\min \left\{\tau_{1}, \tau_{2}, \tau_{3}\right\},
$$


where

$$
\begin{gathered}
\tau_{1}=\min \left\{r\left(\begin{array}{ccc}
O & O & C_{4} \\
O & O & C_{2} \\
B_{3} & B_{1} & A
\end{array}\right), r\left(\begin{array}{ccc}
O & C_{4} & O \\
B_{3} & A & B_{2}
\end{array}\right), r\left(\begin{array}{cc}
O & C_{4} \\
B_{3} & A \\
O & C_{1}
\end{array}\right)\right\}, \\
\tau_{2}=\min \left\{r\left(\begin{array}{ccc}
O & C_{2} & O \\
B_{1} & A & B_{4}
\end{array}\right), r\left(A B_{2} B_{4}\right), r\left(\begin{array}{cc}
A & B_{4} \\
C_{1} & O
\end{array}\right)\right\}, \\
\tau_{3}=\min \left\{r\left(\begin{array}{cc}
O & C_{2} \\
B_{1} & A \\
O & C_{3}
\end{array}\right), r\left(\begin{array}{cc}
A & B_{2} \\
C_{3} & O
\end{array}\right), r\left(\begin{array}{c}
A \\
C_{1} \\
C_{3}
\end{array}\right)\right\} .
\end{gathered}
$$

Proof. In fact, we can write $B_{1}=B_{2} X, B_{3}=B_{4} Y, C_{2}=Z C_{1}$, and $C_{4}=W C_{3}$ under the hypotheses of Theorem 2.3. In this case, we have

$$
\begin{aligned}
& r\left(\begin{array}{cccc}
O & O & C_{4} & O \\
B_{3} & B_{1} & A & B_{2}
\end{array}\right)=r\left(\begin{array}{ccc}
O & C_{4} & O \\
B_{3} & A & B_{2}
\end{array}\right), r\left(\begin{array}{cc}
O & C_{4} \\
O & C_{2} \\
B_{3} & A \\
O & C_{1}
\end{array}\right)=r\left(\begin{array}{cc}
O & C_{4} \\
B_{3} & A \\
O & C_{1}
\end{array}\right), \\
& r\left(\begin{array}{ccc}
O & O & O \\
O & O & C_{2} \\
B_{3} & B_{1} & A
\end{array}\right)=r\left(\begin{array}{ccc}
O & O & C_{4} \\
O & O & Z C_{1} \\
B_{3} & B_{2} X & A
\end{array}\right) \leq r\left(\begin{array}{ccc}
O & C_{4} & O \\
B_{3} & A & B_{2} \\
O & C_{1} & O
\end{array}\right)
\end{aligned}
$$

and

$$
\begin{aligned}
& r\left(\begin{array}{llll}
B_{1} & A & B_{2} & B_{4} \\
O & C_{3} & O & O
\end{array}\right)=r\left(\begin{array}{ccc}
A & B_{2} & B_{4} \\
C_{3} & O & O
\end{array}\right), r\left(\begin{array}{cc}
C_{2} & O \\
A & B_{4} \\
C_{1} & O \\
C_{3} & O
\end{array}\right)=r\left(\begin{array}{cc}
A & B_{4} \\
C_{1} & O \\
C_{3} & O
\end{array}\right), \\
& r\left(\begin{array}{ccc}
O & C_{2} & O \\
B_{1} & A & B_{4} \\
O & C_{3} & O
\end{array}\right)=r\left(\begin{array}{ccc}
O & Z C_{1} & O \\
B_{2} X & A & B_{4} \\
O & C_{3} & O
\end{array}\right) \leq r\left(\begin{array}{lll}
A & B_{2} & B_{4} \\
C_{1} & O & O \\
C_{3} & O & O
\end{array}\right)
\end{aligned}
$$

and

$$
\begin{aligned}
& r\left(B_{3} B_{1} A B_{2} B_{4}\right)=r\left(\begin{array}{lll}
A & B_{2} & B_{4}
\end{array}\right)=r\left(\begin{array}{ccc}
O & C_{2} & O \\
B_{3} & A & B_{4} \\
O & C_{1} & O
\end{array}\right)=r\left(\begin{array}{cc}
A & B_{4} \\
C_{1} & O
\end{array}\right), \\
& r\left(\begin{array}{cccc}
O & O & C_{2} & O \\
B_{3} & B_{1} & A & B_{4}
\end{array}\right)=r\left(\begin{array}{cccc}
O & O & Z C_{1} & O \\
B_{3} & B_{2} X & A & B_{4}
\end{array}\right) \leq r\left(\begin{array}{cccc}
B_{3} & A & B_{2} & B_{4} \\
O & C_{1} & O & O
\end{array}\right) \\
& r\left(\begin{array}{cccc}
O & O & C_{2} & O \\
B_{3} & B_{1} & A & B_{4}
\end{array}\right)=r\left(\begin{array}{ccc}
O & C_{2} & O \\
B_{1} & A & B_{4}
\end{array}\right)
\end{aligned}
$$

and

$$
\begin{aligned}
& r\left(\begin{array}{lll}
O & C_{4} & O \\
B_{1} & A & B_{2} \\
O & C_{3} & O
\end{array}\right)=r\left(\begin{array}{cc}
A & B_{2} \\
C_{3} & O
\end{array}\right), r\left(\begin{array}{l}
C_{4} \\
C_{2} \\
A \\
C_{1} \\
C_{3}
\end{array}\right)=r\left(\begin{array}{l}
A \\
C_{1} \\
C_{3}
\end{array}\right), r\left(\begin{array}{cc}
O & C_{4} \\
O & C_{2} \\
B_{1} & A \\
O & C_{3}
\end{array}\right)=r\left(\begin{array}{cc}
O & C_{2} \\
B_{1} & A \\
O & C_{3}
\end{array}\right), \\
& r\left(\begin{array}{cc}
O & C_{4} \\
O & C_{2} \\
B_{1} & A \\
O & C_{3}
\end{array}\right)=r\left(\begin{array}{cc}
O & C_{4} \\
O & Z C_{1} \\
B_{2} X & A \\
O & C_{3}
\end{array}\right) \leq r\left(\begin{array}{cc}
C_{4} & O \\
A & B_{2} \\
C_{1} & O \\
C_{3} & O
\end{array}\right)
\end{aligned}
$$


Combining (5) with (13)-(16) yields (12).

Corollary 2.4 Let $P\left(V_{1}, \quad V_{2}, V_{3}, V_{4}\right)$ be given as (4) and let $R\left(B_{1}\right) \subseteq R\left(B_{2}\right), R\left(B_{3}\right) \subseteq R\left(B_{4}\right), R\left(C_{2}^{*}\right) \subseteq R\left(C_{1}^{*}\right), R\left(C_{4}^{*}\right) \subseteq R\left(C_{3}^{*}\right)$ then the matrix equation $A=B_{1} V_{1} C_{1}+B_{2} V_{2} C_{2}+B_{3} V_{3} C_{3}+B_{4} V_{4} C_{4}$ holds for any $V_{1}, V_{2}, V_{3}$, and $V_{4}$ if and only if $\tau_{1}=O$ or $\tau_{2}=O$ or $\tau_{3}=O$.

In the rest of this section, we will find the minimal rank of the linear matrix expression $P\left(V_{1}, V_{2}, V_{3}, V_{4}\right)$ in (4), with respect to four variant matrices $V_{i} \in C^{p_{i} \times q_{i}}, i=1,2,3,4$, when $P\left(V_{1}, V_{2}, V_{3}, V_{4}\right)$ satisfy some restrictions.

Theorem 2.5 Let $P\left(V_{1}, \quad V_{2}, V_{3}, V_{4}\right)$ be given as (4) and let $R\left(B_{1}\right) \subseteq R\left(B_{2}\right), R\left(B_{3}\right) \subseteq R\left(B_{4}\right), R\left(C_{2}^{*}\right) \subseteq R\left(C_{1}^{*}\right), R\left(C_{4}^{*}\right) \subseteq R\left(C_{3}^{*}\right)$. Then

$$
\begin{aligned}
& \min _{V_{1}, V_{2}, V_{3}, V_{4}} r\left(P\left(V_{1}, V_{2}, V_{3}, V_{4}\right)\right) \\
& =r\left(\begin{array}{lll}
A & B_{2} & B_{4}
\end{array}\right)+r\left(\begin{array}{cc}
A & B_{4} \\
C_{1} & O
\end{array}\right)+r\left(\begin{array}{ccc}
O & C_{2} & O \\
B_{1} & A & B_{4}
\end{array}\right)+r\left(\begin{array}{cc}
A & B_{2} \\
C_{3} & O
\end{array}\right)+r\left(\begin{array}{cc}
O & C_{2} \\
B_{1} & A \\
O & C_{3}
\end{array}\right) \\
& +r\left(\begin{array}{l}
A \\
C_{1} \\
C_{3}
\end{array}\right)-r\left(\begin{array}{ccc}
B_{1} & A & B_{4} \\
O & C_{1} & O
\end{array}\right)-r\left(\begin{array}{ccc}
O & C_{2} & O \\
B_{2} & A & B_{4}
\end{array}\right)-r\left(\begin{array}{cc}
B_{1} & A \\
O & C_{1} \\
O & C_{3}
\end{array}\right)-r\left(\begin{array}{cc}
C_{2} & O \\
A & B_{2} \\
C_{3} & O
\end{array}\right) \\
& +\max \left\{r\left(\begin{array}{ccc}
O & C_{4} & O \\
B_{3} & A & B_{2}
\end{array}\right)+r\left(\begin{array}{cc}
O & C_{4} \\
B_{3} & A \\
O & C_{1}
\end{array}\right)+r\left(\begin{array}{ccc}
O & O & C_{4} \\
O & O & C_{2} \\
B_{3} & B_{1} & A
\end{array}\right)-r\left(\begin{array}{ccc}
O & O & C_{4} \\
O & O & C_{1} \\
B_{3} & B_{1} & A
\end{array}\right)\right. \\
& -r\left(\begin{array}{ccc}
O & O & C_{4} \\
O & O & C_{2} \\
B_{3} & B_{2} & A
\end{array}\right)-\beta_{1}-\beta_{2}, r\left(\begin{array}{ccc}
A & B_{2} & B_{4} \\
C_{3} & O & O
\end{array}\right)+r\left(\begin{array}{cc}
A & B_{4} \\
C_{1} & O \\
C_{3} & O
\end{array}\right) \\
& \left.+r\left(\begin{array}{ccc}
O & C_{2} & O \\
B_{1} & A & B_{4} \\
O & C_{3} & O
\end{array}\right)-r\left(\begin{array}{ccc}
B_{1} & A & B_{4} \\
O & C_{1} & O \\
O & C_{3} & O
\end{array}\right)-r\left(\begin{array}{ccc}
C_{2} & O & O \\
A & B_{2} & B_{4} \\
C_{3} & O & O
\end{array}\right)-2 \beta_{3}\right\},
\end{aligned}
$$

where

$$
\begin{aligned}
& \beta_{1}=\min \left\{r\left(\begin{array}{ccc}
O & O & C_{2} \\
B_{3} & B_{1} & A \\
O & O & C_{3}
\end{array}\right), r\left(\begin{array}{ccc}
B_{3} & A & B_{2} \\
O & C_{3} & O
\end{array}\right), r\left(\begin{array}{cc}
B_{3} & A \\
O & C_{1} \\
O & C_{3}
\end{array}\right)\right\}, \\
& \beta_{2}=\min \left\{r\left(\begin{array}{ccc}
O & C_{4} & O \\
O & C_{2} & O \\
B_{1} & A & B_{4}
\end{array}\right), r\left(\begin{array}{ccc}
C_{4} & O & O \\
A & B_{2} & B_{4}
\end{array}\right), r\left(\begin{array}{cc}
C_{4} & O \\
A & B_{4} \\
C_{1} & O
\end{array}\right)\right\}, \\
& \beta_{3}=\min \left\{r\left(\begin{array}{ccc}
O & C_{2} & O \\
B_{1} & A & B_{4} \\
O & C_{3} & O
\end{array}\right), r\left(\begin{array}{ccc}
A & B_{2} & B_{4} \\
C_{3} & O & O
\end{array}\right), r\left(\begin{array}{cc}
A & B_{4} \\
C_{1} & O \\
C_{3} & O
\end{array}\right)\right\} .
\end{aligned}
$$

Proof. From the proof of Theorem 2.1, it is easy to verify that the minimal rank of $P$ $\left(V_{1}, V_{2}, V_{3}, V_{4}\right)$ in (4) can be expressed as

$$
\min _{V_{1}, V_{2}, V_{3}, V_{4}} r\left(P\left(V_{1}, V_{2}, V_{3}, V_{4}\right)\right)=\min _{V_{1}, V_{2}, V_{3}, V_{4}} r(T)-\sum_{i=1}^{4} p_{i}-\sum_{i=1}^{4} q_{i},
$$

where $T, S, E_{i}, p_{i}$ and $q_{i}, i=1,2,3,4$, are given as the proof of Theorem 2.1. Then applying the formula (2) in Lemma 1.1 to matrix $T$, we have 


$$
\begin{aligned}
\min _{V_{1}, V_{2}, V_{3}, V_{4}} r(T)= & \min _{V_{3}, V_{4}} r\left(\begin{array}{ccc}
O & E_{2} & -V_{4} \\
E_{1} & S & E_{3} \\
-V_{3} & E_{4} & O
\end{array}\right)=r\left(\begin{array}{lll}
E_{1} & S & E_{3}
\end{array}\right)+r\left(\begin{array}{c}
E_{2} \\
S \\
E_{4}
\end{array}\right) \\
+ & \max \left\{r\left(\begin{array}{cc}
O & E_{2} \\
E_{1} & S
\end{array}\right)-r\left(\begin{array}{c}
E_{2} \\
S
\end{array}\right)-r\left(\begin{array}{ll}
E_{1} & S
\end{array}\right),\right. \\
& \left.r\left(\begin{array}{cc}
S & E_{3} \\
E_{4} & O
\end{array}\right)-r\left(\begin{array}{c}
S \\
E_{4}
\end{array}\right)-r\left(\begin{array}{ll}
S & E_{3}
\end{array}\right)\right\} .
\end{aligned}
$$

In this case, we derive from (19) that

$$
\begin{aligned}
& \min _{V_{1}, V_{2}, V_{3}, V_{4}} r(T)=\min _{V_{1}, V_{2}} r\left(\begin{array}{lll}
E_{1} & S & E_{3}
\end{array}\right)+\min _{V_{1}, V_{2}} r\left(\begin{array}{c}
E_{2} \\
S \\
E_{4}
\end{array}\right) \\
& +\max \left\{\min _{V_{1}, V_{2}} r\left(\begin{array}{cc}
O & E_{2} \\
E_{1} & S
\end{array}\right)-\min _{V_{1}, V_{2}} r\left(\begin{array}{c}
E_{2} \\
S
\end{array}\right)-\min _{V_{1}, V_{2}} r\left(\begin{array}{ll}
E_{1} & S
\end{array}\right),\right. \\
& \left.\min _{V_{1}, V_{2}} r\left(\begin{array}{cc}
S & E_{3} \\
E_{4} & O
\end{array}\right)-\min _{V_{1}, V_{2}} r\left(\begin{array}{c}
S \\
E_{4}
\end{array}\right)-\min _{V_{1}, V_{2}} r\left(\begin{array}{ll}
S & E_{3}
\end{array}\right)\right\} \text {. }
\end{aligned}
$$

Again applying the formula (2) in Lemma 1.1, we have

$$
\begin{aligned}
& \min _{V_{1}, V_{2}} r\left(E_{1} S E_{3}\right)=q_{4}+q_{3}+\min _{V_{1}, V_{2}} r\left(S_{3}\right) \\
& =\sum_{i=1}^{4} q_{i}+p_{1}+p_{2}+r\left(\begin{array}{lllll}
B_{3} & B_{1} & A & B_{2} & B_{4}
\end{array}\right)+r\left(\begin{array}{ccc}
O & C_{2} & O \\
B_{3} & A & B_{4} \\
O & C_{1} & O
\end{array}\right) \\
& +\max \left\{r\left(\begin{array}{cccc}
O & O & C_{2} & O \\
B_{3} & B_{1} & A & B_{4}
\end{array}\right)-r\left(\begin{array}{cccc}
O & O & C_{2} & O \\
B_{3} & B_{1} & A & B_{4} \\
O & O & C_{1} & O
\end{array}\right)-r\left(\begin{array}{ccccc}
O & O & C_{2} & O & O \\
B_{3} & B_{1} & A & B_{2} & B_{4}
\end{array}\right),\right. \\
& \left.r\left(\begin{array}{cccc}
B_{3} & A & B_{2} & B_{4} \\
O & C_{1} & O & O
\end{array}\right)-r\left(\begin{array}{cccc}
O & C_{2} & O & O \\
B_{3} & A & B_{2} & B_{4} \\
O & C_{1} & O & O
\end{array}\right)-r\left(\begin{array}{ccccc}
B_{3} & B_{1} & A & B_{2} & B_{4} \\
O & O & C_{1} & O & O
\end{array}\right)\right\},
\end{aligned}
$$

where $S_{3}$ is given as the Equation (7) of the proof of Theorem 2.1. Since $B_{1}=B_{2} X$, $B_{3}=B_{4} Y, C_{2}=Z C_{1}$, and $C_{4}=W C_{3},(21)$ is reduced to

$$
\begin{aligned}
& \min _{V_{1}, V_{2}} r\left(E_{1} S E_{3}\right) \\
& =\sum_{i=1}^{4} q_{i}+p_{1}+p_{2}+r\left(\begin{array}{lll}
A & B_{2} & B_{4}
\end{array}\right)+r\left(\begin{array}{cc}
A & B_{4} \\
C_{1} & O
\end{array}\right) \\
& +\max \left\{r\left(\begin{array}{ccc}
O & C_{2} & O \\
B_{1} & A & B_{4}
\end{array}\right)-r\left(\begin{array}{ccc}
B_{1} & A & B_{4} \\
O & C_{1} & O
\end{array}\right)-r\left(\begin{array}{ccc}
C_{2} & O & O \\
A & B_{2} & B_{4}
\end{array}\right),-r\left(\begin{array}{ccc}
A & B_{2} & B_{4} \\
C_{1} & O & O
\end{array}\right)\right\} \\
& =\sum_{i=1}^{4} q_{i}+p_{1}+p_{2}+r\left(\begin{array}{lll}
A & B_{2} & B_{4}
\end{array}\right)+r\left(\begin{array}{cc}
A & B_{4} \\
C_{1} & O
\end{array}\right)+r\left(\begin{array}{ccc}
O & C_{2} & O \\
B_{1} & A & B_{4}
\end{array}\right)-r\left(\begin{array}{ccc}
B_{1} & A & B_{4} \\
O & C_{1} & O
\end{array}\right) \\
& -r\left(\begin{array}{ccc}
C_{2} & O & O \\
A & B_{2} & B_{4}
\end{array}\right) .
\end{aligned}
$$

The last equality holds, since the well-known Frobenius rank inequality $r(A B C) \geq r$ $(A B)+r(B C)-r(B)$, then 


$$
\begin{aligned}
r\left(\begin{array}{ccc}
O & C_{2} & O \\
B_{1} & A & B_{4}
\end{array}\right)= & r\left(\begin{array}{ccc}
O & Z C_{1} & O \\
B_{2} X & A & B_{4}
\end{array}\right) \\
= & r\left(\left(\begin{array}{cc}
Z & O \\
O & I
\end{array}\right)\left(\begin{array}{ccc}
O & C_{1} & O \\
B_{2} & A & B_{4}
\end{array}\right)\left(\begin{array}{ccc}
X & O & O \\
O & I & O \\
O & O & I
\end{array}\right)\right) \\
\geq & r\left(\left(\begin{array}{cc}
Z & O \\
O & I
\end{array}\right)\left(\begin{array}{ccc}
O & C_{1} & O \\
B_{2} & A & B_{4}
\end{array}\right)\right)+r\left(\left(\begin{array}{ccc}
O & C_{1} & O \\
B_{2} & A & B_{4}
\end{array}\right)\left(\begin{array}{ccc}
X & O & O \\
O & I & O \\
O & O & I
\end{array}\right)\right) \\
& \left.-r\left(\begin{array}{ccc}
O & C_{1} & O \\
B_{2} & A & B_{4}
\end{array}\right)\right) \\
= & r\left(\begin{array}{ccc}
O & C_{2} & O \\
B_{2} & A & B_{4}
\end{array}\right)+r\left(\begin{array}{ccc}
O & C_{1} & O \\
B_{1} & A & B_{4}
\end{array}\right)-r\left(\begin{array}{ccc}
O & C_{1} & O \\
B_{2} & A & B_{4}
\end{array}\right)
\end{aligned}
$$

With the similar method, we also have

$$
\begin{aligned}
& \min _{V_{1}, V_{2}} r\left(\begin{array}{c}
E_{2} \\
S \\
E_{4}
\end{array}\right)=\sum_{i=1}^{4} p_{i}+q_{1}+q_{2}+r\left(\begin{array}{c}
A \\
C_{1} \\
C_{3}
\end{array}\right)+r\left(\begin{array}{cc}
A & B_{2} \\
C_{3} & O
\end{array}\right)+r\left(\begin{array}{cc}
O & C_{2} \\
B_{1} & A \\
O & C_{3}
\end{array}\right) \\
& -r\left(\begin{array}{ll}
B_{1} & A \\
O & C_{1} \\
O & C_{3}
\end{array}\right)-r\left(\begin{array}{cc}
C_{2} & O \\
A & B_{2} \\
C_{3} & O
\end{array}\right) \\
& \min _{V_{1}, V_{2}} r\left(\begin{array}{cc}
O & E_{2} \\
E_{1} & S
\end{array}\right)=p_{1}+p_{2}+p_{4}+q_{1}+q_{2}+q_{3}+r\left(\begin{array}{ccc}
O & C_{4} & O \\
B_{3} & A & B_{2}
\end{array}\right)+r\left(\begin{array}{cc}
O & C_{4} \\
B_{3} & A \\
O & C_{1}
\end{array}\right) \\
& +r\left(\begin{array}{ccc}
O & O & C_{4} \\
O & O & C_{2} \\
B_{3} & B_{1} & A
\end{array}\right)-r\left(\begin{array}{ccc}
O & O & C_{4} \\
O & O & C_{1} \\
B_{3} & B_{1} & A
\end{array}\right)-r\left(\begin{array}{ccc}
O & O & C_{4} \\
O & O & C_{2} \\
B_{3} & B_{2} & A
\end{array}\right) \\
& \min _{V_{1}, V_{2}} r\left(\begin{array}{cc}
S & E_{3} \\
E_{4} & O
\end{array}\right)=q_{1}+q_{2}+q_{4}+p_{1}+p_{2}+p_{3}+r\left(\begin{array}{ccc}
A & B_{2} & B_{4} \\
C_{3} & O & O
\end{array}\right)+r\left(\begin{array}{cc}
A & B_{4} \\
C_{1} & O \\
C_{3} & O
\end{array}\right) \\
& +r\left(\begin{array}{lll}
O & C_{2} & O \\
B_{1} & A & B_{4} \\
O & C_{3} & O
\end{array}\right)-r\left(\begin{array}{ccc}
B_{1} & A & B_{4} \\
O & C_{1} & O \\
O & C_{3} & O
\end{array}\right)-r\left(\begin{array}{ccc}
C_{2} & O & O \\
A & B_{2} & B_{4} \\
C_{3} & O & O
\end{array}\right) .
\end{aligned}
$$

On the other hand, by the formula (1) in Lemma 1.1, we have

$$
\begin{gathered}
\min _{V_{1}, V_{2}} r\left(\begin{array}{c}
E_{2} \\
S
\end{array}\right)=p_{4}+p_{1}+p_{2}+q_{1}+q_{2}+\min \left\{r\left(\begin{array}{ccc}
O & O & C_{2} \\
B_{3} & B_{1} & A \\
O & O & C_{3}
\end{array}\right),\right. \\
r\left(\begin{array}{ccc}
O & C_{4} & O \\
B_{3} & A & B_{2} \\
O & C_{3} & O
\end{array}\right), \\
\left.r\left(\begin{array}{ll}
B_{3} & A \\
O & C_{1} \\
O & C_{3}
\end{array}\right)\right\} .
\end{gathered}
$$




$$
\begin{aligned}
& \max _{V_{1}, V_{2}} r\left(\begin{array}{c}
S \\
E_{4}
\end{array}\right)=p_{3}+p_{1}+p_{2}+q_{1}+q_{2}+\min \left\{r\left(\begin{array}{ccc}
O & C_{2} & O \\
B_{1} & A & B_{4} \\
O & C_{3} & O
\end{array}\right), r\left(\begin{array}{ccc}
C_{4} & O & O \\
A & B_{2} & B_{4} \\
C_{3} & O & O
\end{array}\right),\right. \\
& \left.r\left(\begin{array}{cc}
A & B_{4} \\
C_{1} & O \\
C_{3} & O
\end{array}\right)\right\} \\
& \max _{V_{1}, V_{2}} r\left(\begin{array}{ll}
E_{1} & S
\end{array}\right)=q_{3}+p_{1}+p_{2}+q_{1}+q_{2}+\min \left\{r\left(\begin{array}{ccc}
O & C_{4} & O \\
O & C_{2} & O \\
B_{1} & A & B_{4}
\end{array}\right), r\left(\begin{array}{ccc}
C_{4} & O & O \\
A & B_{2} & B_{4}
\end{array}\right),\right. \\
& \left.r\left(\begin{array}{cc}
C_{4} & O \\
A & B_{4} \\
C_{1} & O
\end{array}\right)\right\} \\
& \max _{V_{1}, V_{2}} r\left(\begin{array}{ll}
S & E_{3}
\end{array}\right)=q_{3}+p_{1}+p_{2}+q_{1}+q_{2}+\min \left\{r\left(\begin{array}{ccc}
O & C_{2} & O \\
B_{1} & A & B_{4} \\
O & C_{3} & O
\end{array}\right), r\left(\begin{array}{ccc}
A & B_{2} & B_{4} \\
C_{3} & O & O
\end{array}\right),\right. \\
& \left.r\left(\begin{array}{cc}
A & B_{4} \\
C_{1} & O \\
C_{3} & O
\end{array}\right)\right\}
\end{aligned}
$$

Contrasting (18), (20) and (22)-(29) yields (17).

Corollary 2.6 Let $P\left(V_{1}, V_{2}, V_{3}, V_{4}\right)$ be given as (4) and let $R\left(B_{1}\right) \subseteq R\left(B_{2}\right), R\left(B_{3}\right) \subseteq R$ $\left(B_{4}\right), R\left(B_{1}\right) \subseteq R\left(B_{2}\right), R\left(B_{3}\right) \subseteq R\left(B_{4}\right), R\left(C_{2}^{*}\right) \subseteq R\left(C_{1}^{*}\right), R\left(C_{4}^{*}\right) \subseteq R\left(C_{3}^{*}\right)$. Then the matrix equation $A=B_{1} V_{1} C_{1}+B_{2} V_{2} C_{2}+B_{3} V_{3} C_{3}+B_{4} V_{4} C_{4}$ is consistent if and only if the right side of (17) is zero.

\section{Some applications to generalized Schur complement and partial matrix}

As direct applications of the results in Section 2, we determine in this section the maximal and minimal ranks of the generalized Schur complement $A-B M^{(1)} C-D N^{(1)} G$ and the partial matrix $\left(A B M^{(1)} C D N^{(1)} G\right)$ with respect to two variant matrices $M^{(1)} L M\{1\}$ and $N^{(1)}\lfloor N\{1\}$.

Theorem 3.1 Let $A L C^{m \times n}, B L C^{m \times p}, C L C^{q \times n}, D L C^{m \times s}, G L C^{t \times n}, M L C^{q \times}$ $p$, and $N L C^{t \times s}$.

Then

$$
\max _{M^{(1)} \in M\{1\}, N^{(1)} \in N\{1\}} r\left(A-B M^{(1)} C-D N^{(1)} G\right)=\min \left\{\widehat{T_{1}}, \widehat{T_{2}}, \widehat{T_{3}}\right\},
$$

where

$$
\begin{gathered}
\widehat{T_{1}=\min }\left\{r\left(\begin{array}{lll}
A & D & B \\
G & N & O \\
C & O & M
\end{array}\right)-r(M)-r(N), r\left(\begin{array}{lll}
A & D & B \\
G & N & O
\end{array}\right)-r(N), r\left(\begin{array}{ll}
A & D \\
G & N \\
C & O
\end{array}\right)-r(N)\right\}, \\
\widehat{T_{2}}=\min \left\{\left(\begin{array}{lll}
A & B & D \\
C & M & O
\end{array}\right)-r(M), r\left(\begin{array}{lll}
A & B & D
\end{array}\right), r\left(\begin{array}{ll}
A & D \\
C & O
\end{array}\right)\right\}, \\
\widehat{T_{3}}=\min \left\{r\left(\begin{array}{ll}
A & B \\
C & M \\
G & O
\end{array}\right)-r(M), r\left(\begin{array}{l}
A \\
C \\
G
\end{array}\right), r\left(\begin{array}{ll}
A & B \\
G & O
\end{array}\right)\right\} .
\end{gathered}
$$


Proof. Applying Lemma 1.2, we have

$$
M^{(1)}=M^{\dagger}+F_{M} W_{1}+W_{2} E_{M}
$$

and

$$
N^{(1)}=N^{\dagger}+F_{N} W_{3}+W_{4} E_{N}
$$

where $W_{i}, i=1,2,3,4$ are arbitrary, $E_{M}=I_{q}-M M^{\dagger}$ and $F_{M}=I_{p}-M^{\dagger} M$. Substituting the Equation (31) and Equation (32) into the generalized Schur complement $A-B M^{(1)}$ $C-D N^{(1)} G$ yields

$$
A-B M^{(1)} C-D N^{(1)} G=A_{1}-B F_{M} W_{1} C-B W_{2} E_{M} C-D F_{N} W_{3} G-D W_{4} E_{N} G
$$

where $A_{1}=A-B M^{\dagger} C-D N^{\dagger} G$.

In fact $A_{1}-B F_{M} W_{1} C-B W_{2} E_{M} C-D F_{N} W_{3} G-D W_{4} E_{N} G$ is a special case of the matrix expression $P\left(V_{1}, V_{2}, V_{3}, V_{4}\right)$, and $R\left(B F_{M}\right) \subseteq R(B), R\left(D F_{N}\right) \subseteq R(D), R\left(\left(E_{M} C\right)^{*}\right) \subseteq R$ $\left(C^{*}\right), R\left(\left(E_{N} G\right)^{*}\right) \subseteq R\left(G^{*}\right)$. In this case, from the formula (12) in Theorem 2.3, we have

$$
\begin{aligned}
& \max _{M^{(1)} \in M\{1\}, N^{(1)} \in N\{1\}} r\left(A-B M^{(1)} C-D N^{(1)} G\right) \\
& =\max _{W_{1}, W_{2}, W_{3}, W_{4}} r\left(A_{1}-B F_{M} W_{1} C-B W_{2} E_{M} C-D F_{N} W_{3} G-D W_{4} E_{N} G\right) \\
& =\min \left\{T_{1}^{\prime}, T_{2}^{\prime}, T_{3}^{\prime}\right\},
\end{aligned}
$$

where

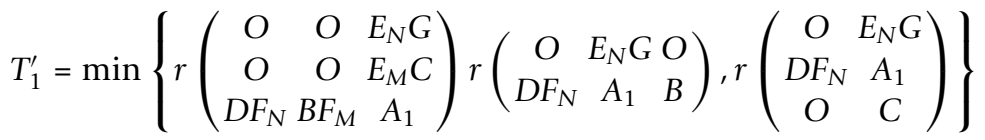

$$
\begin{aligned}
& T_{2}^{\prime}=\min \left\{r\left(\begin{array}{lll}
O & E_{M} C & O \\
B F_{M} & A_{1} & D
\end{array}\right), r\left(A_{1} B B\right), r\left(\begin{array}{ll}
A_{1} & D \\
C & O
\end{array}\right)\right\} \\
& T_{3}^{\prime}=\min \left\{r\left(\begin{array}{cc}
O & E_{M} C \\
B F_{M} & A_{1} \\
O & G
\end{array}\right), r\left(\begin{array}{cc}
A_{1} & B \\
G & O
\end{array}\right), r\left(\begin{array}{c}
A_{1} \\
C \\
G
\end{array}\right)\right\}
\end{aligned}
$$

For $T_{1}{ }^{\prime}$, simplifying the ranks of matrices by Lemma 1.3 and block Gaussian elimination, we find that:

$$
\begin{aligned}
& r\left(\begin{array}{ccc}
O & O & E_{N} G \\
O & O & E_{M} C \\
D F_{N} & B F_{M} & A_{1}
\end{array}\right)=\left(\begin{array}{ccc}
A_{1} & D F_{N} & B F_{M} \\
E_{M} G & O & O \\
E_{M} C & O & O
\end{array}\right) \\
& =r\left(\begin{array}{llllll}
A_{1} & D & B & O & O & O \\
O & N & O & O & O & O \\
O & O & M & O & O & O \\
G & O & O & O & N & O \\
C & O & O & O & O & M
\end{array}\right)-2 r(N)-2 r(M) \\
& =r\left(\begin{array}{lll}
A & D & B \\
G & N & O \\
C & O & M
\end{array}\right)-r(N)-r(M),
\end{aligned}
$$




$$
\begin{aligned}
& r\left(\begin{array}{ccc}
O & E_{N} G & O \\
D F_{N} & A_{1} & B
\end{array}\right)=r\left(\begin{array}{ccc}
A_{1} & D F_{N} & B \\
E_{N} G & O & O
\end{array}\right)=r\left(\begin{array}{cccc}
A_{1} & D & B & O \\
O & N & O & O \\
G & O & O & N
\end{array}\right)-2 r(N) \\
& =r\left(\begin{array}{lll}
A & D & B \\
G & N & O
\end{array}\right)-r(N), \\
& r\left(\begin{array}{cc}
O & E_{N} G \\
D F_{N} & A_{1} \\
O & C
\end{array}\right)=\left(\begin{array}{cccc}
O & G & N & O \\
O & C & O & M \\
D & A_{1} & O & O \\
N & O & O & O \\
O & C & O & O
\end{array}\right)-2 r(N)=r\left(\begin{array}{cc}
A & D \\
G & N \\
C & O
\end{array}\right)-r(N)
\end{aligned}
$$

Combining the rank equalities (35), (36) with (37), we have $T_{1}^{\prime}=\widehat{T_{1}}$.

By the similar approach, we also have $T_{2}^{\prime}=\widehat{T_{2}}$ and $T_{3}^{\prime}=\widehat{T_{3}}$. Then we have complete the proof of theorem.

Corollary 3.2 Let $A L C^{m \times n}, B L C^{m \times p}, C L C^{q \times n}, D L C^{m \times s}, G L C^{t \times n}, M L C^{q \times}$ ${ }^{p}$, and $N L C^{t \times s}$. Then the identity $A=B M^{(1)} C+D N^{(1)} G$ holds for every $M^{(1)} L M\{1\}$ and $N^{(1)} L N\{1\}$ if and only if $\widehat{T_{1}}=O$ or $\widehat{T_{2}}=O$ or $\widehat{T_{3}}=O$.

From the proof of Theorem 3.1, we known that $A-B M^{(1)} C-D N^{(1)} G=A_{1}$ $B F_{m} W_{1} C-B W_{2} E_{m} C-D F_{N} W_{3} G-D W_{4} E_{N} G$, where $A_{1}=A-B M^{\dagger} C-D N^{\dagger} G$. In this case, $A-B M^{(1)} C-D N^{(1)} G$ is a special case of the matrix expression $P\left(V_{1}, V_{2}, V_{3}, V_{4}\right)$, and $R\left(B F_{M}\right) \subseteq R(B), R\left(D F_{N}\right) \subseteq R(D), R\left(\left(E_{M} C\right)^{*}\right) \subseteq R\left(C^{*}\right), R\left(\left(E_{N} G\right)^{*}\right) \subseteq R\left(G^{*}\right)$. Then from the Theorem 2.5, we have

Theorem 3.3 Let $A L C^{m \times n}, B L C^{m \times p}, C L C^{q \times n}, D L C^{m \times s}, G L C^{t \times n}, M L C^{q \times}$ $p$, and $N L C^{t \times s}$.

Then

$$
\begin{aligned}
& \min _{M^{(1)} \in M\{1\}, N^{(1)} \in N\{1\}} r\left(A-B M^{(1)} C-D N^{(1)} G\right) \\
& =\min _{W_{1}, W_{2}, W_{3}, W_{4}} r\left(A_{1}-B F_{M} W_{1} C-B W_{2} E_{M} C-D F_{N} W_{3} G-D W_{4} E_{N} G\right) \\
& =r\left(\begin{array}{lll}
A & D
\end{array}\right)+r\left(\begin{array}{ll}
A & D \\
C & O
\end{array}\right)+r\left(\begin{array}{lll}
M & C & O \\
B & A & D
\end{array}\right)+r\left(\begin{array}{l}
A \\
C \\
G
\end{array}\right)+r\left(\begin{array}{ll}
A & B \\
G & O
\end{array}\right)+r\left(\begin{array}{ll}
M & C \\
B & A \\
O & G
\end{array}\right)
\end{aligned}
$$

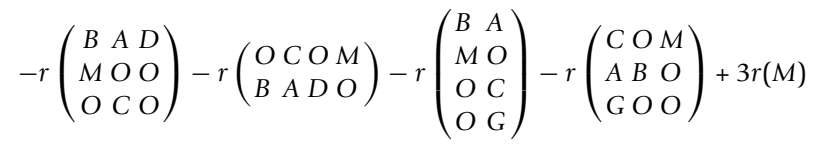

$$
\begin{aligned}
& +\max \left\{r\left(\begin{array}{lll}
N & G & O \\
D & A & B
\end{array}\right)+r\left(\begin{array}{lll}
O & G & N \\
B & A & O \\
O & C & O
\end{array}\right)+r\left(\begin{array}{lll}
N & O & G \\
O & M & C \\
D & B & A
\end{array}\right)-r\left(\begin{array}{lll}
N & O & G \\
O & O & C \\
D & B & A \\
O & M & O
\end{array}\right)\right. \\
& -r\left(\begin{array}{cccc}
N & O & G & O \\
O & O & C & M \\
D & B & A & O
\end{array}\right)+r(N)-\delta_{1}-\delta_{2} \\
& r\left(\begin{array}{ccc}
A & B & D \\
G & O & O
\end{array}\right)+r\left(\begin{array}{ll}
A & B \\
C & O \\
G & O
\end{array}\right)+r\left(\begin{array}{ccc}
M & C & O \\
B & A & D \\
O & G & O
\end{array}\right)-r\left(\begin{array}{lll}
B & A & D \\
M & O & O \\
O & C & O \\
O & G & O
\end{array}\right) \\
& \left.-r\left(\begin{array}{cccc}
C & O & O & M \\
A & B & D & O \\
G & O & O & O
\end{array}\right)-2 \delta_{3}\right\}
\end{aligned}
$$


where

$$
\begin{aligned}
& \delta_{1}=\min \left\{r\left(\begin{array}{ccc}
O & M & C \\
D & B & A \\
N & O & O \\
O & O & G
\end{array}\right)-r(M), r\left(\begin{array}{ccc}
D & A & B \\
N & O & O \\
O & G & O
\end{array}\right), r\left(\begin{array}{ll}
D & A \\
N & O \\
O & C \\
O & G
\end{array}\right)\right\}, \\
& \delta_{2}=\min \left\{r\left(\begin{array}{cccc}
O & G & O & N \\
M & C & O & O \\
B & A & D & O
\end{array}\right)-r(M), r\left(\begin{array}{cccc}
G & O & O & N \\
A & B & D & O
\end{array}\right), r\left(\begin{array}{lll}
G & O & N \\
A & D & O \\
C & O & O
\end{array}\right)\right\} \text {, } \\
& \delta_{3}=\min \left\{r\left(\begin{array}{ccc}
M & C & O \\
B & A & D \\
O & G & O
\end{array}\right)-r(M), r\left(\begin{array}{lll}
A & B & D \\
G & O & O
\end{array}\right), r\left(\begin{array}{ll}
A & D \\
C & O \\
G & O
\end{array}\right)\right\} \text {. }
\end{aligned}
$$

Corollary 3.4 Let $A L C^{m \times n}, B L C^{m \times p}, C L C^{q \times n}, D L C^{m \times s}, G L C^{t \times n}, M L C^{q \times}$ ${ }^{p}$, and $N L C^{t \times s}$. then the identity $A=B M^{(1)} C+D N^{(1)} G$ is consistent if and only if the right side of (38) is zero.

Next, we will determine the maximal and minimal ranks of the partial matrix

$$
\left(\begin{array}{lll}
A & \left.B M^{(1)} C \quad D N^{(1)} G\right)
\end{array}\right.
$$

with respect to $M^{(1)} L M\{1\}$ and $N^{(1)} L N\{1\}$, by applying the results in Section 2 . It is quite obvious that the partial matrix $\left(A B M^{(1)} C D N^{(1)} G\right)$ may be written as

$$
\left(\begin{array}{lll}
A & B M^{(1)} C & D N^{(1)} G
\end{array}\right)=\left(\begin{array}{lll}
A & O & O
\end{array}\right)+B M^{(1)}\left(\begin{array}{lll}
O & C & O
\end{array}\right)+D N^{(1)}\left(\begin{array}{lll}
O & O & G
\end{array}\right) .
$$

Then from (39) and Theorems 2.3 and 3.1, we have

Theorem 3.5 Let $A L C^{m \times n}, B L C^{m \times p}, C L C^{q \times n}, D L C^{m \times s}, G L C^{t \times n}, M L C^{q \times}$ $p$, and $N L C^{t \times s}$.

Then

$$
\max _{M^{(1)} \in M\{1\}, N^{(1)} \in N\{1\}} r\left(A \quad B M^{(1)} C \quad D N^{(1)} G\right)=\min \left\{\tilde{T}_{1}, \tilde{T}_{2}, \tilde{T}_{3}\right\} ，
$$

where

$$
\begin{aligned}
& \tilde{T}_{1}=\min \left\{r\left(\begin{array}{lllll}
A & O & O & D & B \\
O & O & G & N & O \\
O & C & O & O & M
\end{array}\right)-r(M)-r(N), r\left(\begin{array}{cccc}
A & O & D & B \\
O & G & N & O
\end{array}\right)-r(N)\right. \\
& \left.r\left(\begin{array}{lll}
A & O & D \\
O & G & N
\end{array}\right)+r(C)-r(N)\right\} \\
& \tilde{T}_{2}=\min \left\{r\left(\begin{array}{llll}
A & O & B & D \\
O & C & M & O
\end{array}\right)-r(M), r(A B D), r(A D)+r(C)\right\}, \\
& \tilde{T}_{3}=\min \left\{r\left(\begin{array}{lll}
A & O & B \\
O & C & M
\end{array}\right)+r(G)-r(M), r(A B)+r(G), r(A)+r(C)+r(G)\right\} \text {. }
\end{aligned}
$$

Corollary 3.6 Let $A \in C^{m \times n}, B \in C^{m \times p}, C \in C^{q \times n}, D \in C^{m \times s}, G \in C^{t \times n}, M \in$ $C^{q \times p}$, and $N \in C^{t \times s}$. then the inclusion $R\left(B M^{(1)} C+D N^{(1)} G\right) \subseteq R(A)$ holds for every $M^{(1)} L M\{1\}$ and $N^{(1)} L N\{1\}$ if and only if $\tilde{T}_{1}=O$ or $\tilde{T}_{2}=O$ or $\tilde{T}_{3}=O$.

On the other hand, from (39) and Theorems 2.5 and 3.3, we can easily obtain the minimal rank of the partial matrix $\left(A B M^{(1)} C D N^{(1)} G\right)$ with respect to $M^{(1)} L M\{1\}$ and $N^{(1)}\lfloor N\{1\}$. 
Theorem 3.7 Let $A \in C^{m \times n}, B \in C^{m \times p}, C \in C^{q \times n}, D \in C^{m \times s}, G \in C^{t \times n}, M \in$ $C^{q \times p}$, and $N \in C^{t \times s}$.

Then

$$
\begin{aligned}
& \min _{M^{(1)} \in M\{1\}, N^{(1)} \in N\{1\}} r\left(A B M^{(1)} C D N^{(1)} G\right) \\
& =r(A)+r\left(\begin{array}{ll}
A & D
\end{array}\right)+r\left(\begin{array}{lll}
A & B
\end{array}\right)+r\left(\begin{array}{lll}
A & B & D
\end{array}\right)+r\left(\begin{array}{cccc}
M & O & C & O \\
B & A & O & D
\end{array}\right) \\
& -r\left(\begin{array}{ll}
B & A \\
M & O
\end{array}\right)-r\left(\begin{array}{lll}
B & A & D \\
M & O & O
\end{array}\right)-r\left(\begin{array}{lllll}
O & O & C & O & M \\
B & A & O & D & O
\end{array}\right)+3 r(M) \\
& +\max \left\{r\left(\begin{array}{cccc}
N & O & G & O \\
D & A & O & B
\end{array}\right)+r\left(\begin{array}{cccc}
O & O & G & N \\
B & A & O & O
\end{array}\right)+r\left(\begin{array}{ccccc}
N & O & O & O & G \\
O & M & O & C & O \\
D & B & A & O & O
\end{array}\right)\right. \\
& -r\left(\begin{array}{llll}
N & O & O & G \\
D & B & A & O \\
O & M & O & O
\end{array}\right)-r\left(\begin{array}{cccccc}
N & O & O & O & G & O a \\
O & O & O & C & O & M \\
D & B & A & O & O & O
\end{array}\right)+r(N)-\xi_{1}-\xi_{2} \\
& r(G)+r(A D)+r\left(\begin{array}{lll}
A & B & D
\end{array}\right)+r\left(\begin{array}{cccc}
M & O & C & O \\
B & A & O & D \\
O & G & O
\end{array}\right)-r\left(\begin{array}{ccc}
B & A & D \\
M & O & O
\end{array}\right)
\end{aligned}
$$

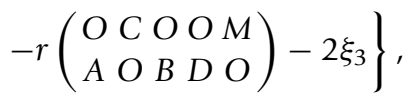

where

$$
\begin{aligned}
& \xi_{1}=\min \left\{r\left(\begin{array}{cccc}
O & M & O & C \\
D & B & A & O \\
N & O & O & O
\end{array}\right)+r(G)-r(M), \quad r\left(\begin{array}{ccc}
D & A & B \\
N & O & O
\end{array}\right)+r(G)\right. \\
& \left.r\left(\begin{array}{ll}
D & A \\
N & O
\end{array}\right)+r(C)+r(G)\right\} \\
& \xi_{2}=\min \left\{r\left(\begin{array}{cccccc}
O & O & O & G & O & N \\
M & O & C & O & O & O \\
B & A & O & O & D & O
\end{array}\right)-r(M), r\left(\begin{array}{ccccc}
O & G & O & O & N \\
A & O & B & D & O
\end{array}\right),\right. \\
& r\left(\begin{array}{lllll}
O & O & G & O & N \\
A & O & O & D & O \\
O & C & O & O & O
\end{array}\right) \\
& \xi_{3}=\min \left\{r\left(\begin{array}{cccc}
M & O & C & O \\
B & A & O & D
\end{array}\right)+r(G)-r(M), r(A B D)+r(G), r(A D)+r(C)+r(G)\right\} \text {. }
\end{aligned}
$$

Corollary 3.8 Let $A \in C^{m \times n}, B \in C^{m \times p}, C \in C^{q \times n}, D L C^{m \times s}, G L C^{t \times n}, M L$ $C^{q \times p}$, and $N L C^{t \times s}$. then there are some $M^{(1)} L M\{1\}$ and $N^{(1)} L N\{1\}$, such that the inclusion $R\left(B M^{(1)} C+D N^{(1)} G\right) \subseteq R(A)$ holds if and only if the right side of (41) is zero.

\section{Acknowledgements}

The authors would likes to thank the Editor-in-Chief and the anonymous referees for their very detailed comments, which greatly improved the presentation of this article. The research of the Z. Xiong was supported by the start-up fund of Wuyi University Jiangmen 529020, Guangdong province, P.R. China and the foundation for High-Level Talents in Guangdong province, P.R. China. The research of the S.Yuan was supported by Guangdong Natural Science Fund of China (No.10452902001005845).

\section{Authors' contributions}

The authors jointly worked on deriving the results. All authors read and approved the final manuscript.

\section{Competing interests}

The authors declare that they have no competing interests. 


\section{References}

1. Penrose, R: A generalized inverse for matrices. Proc Cambridge Philos Soc. 51, 406-413 (1955). doi:10.1017/ S0305004100030401

2. Ben-Israel, A, Greville, TNE: Generalized Inverse: Theory and Applications, Wiley-Interscience, 1974, 2nd edn.SpringerVerlag, New York (2002)

3. Wang, G, Wei, Y, Qiao, S: Generalized Inverses: Theory and Computations. Science Press, Beijing (2004)

4. Braden, HW: The matrix equation $A^{\top} X \pm X^{\top} A=B$. SIAM J Matrix Anal Appl. 20, 295-302 (1998). doi:10.1137/ S0895479897323270

5. Johnson, CR, Whitney, GT: Minimum rank completions. Linear and multilinear Algebra. 28, 271-273 (1991). doi:10.1080/ 03081089108818051

6. Tian, Y: Ranks of solutions of the matrix equation $A X B=$ C. Linear and multilinear Algebra. 51, 111-125 (2003). doi:10.1080/0308108031000114631

7. Bostain, AA, Woerdeman, HJ: Unicity of minimal rank completions for tri-diagonal partial block matrices. Linear Algebra Appl. 325, 23-25 (2001). doi:10.1016/S0024-3795(00)00253-6

8. Cohen, N, Johnson, CR, Rodman, L, Woerdeman, HJ: Ranks of completions of partial matrices. Oper Theory Adv Appl. 40, 165-185 (1989)

9. Marsaglia, G, Styan, GPH: Equalities and inequalities for ranks of matrices. Linear and multilinear Algebra. 2, 269-292 (1974). doi:10.1080/03081087408817070

10. Woerdeman, HJ: Minimal rank completions for block matrices. Linear Algebra Appl. 121, 105-122 (1989)

11. Woerdeman, HJ: Minimal rank completions of partial banded matrices. Linear and multilinear Algebra. 36, 59-69 (1993). doi:10.1080/03081089308818275

12. Davis, C: Completing a matrix so as to minimize its rank. Oper Theory Adv Appl. 29, 87-95 (1988)

13. Johnson, CR: Matrix completion problems: a survey in matrix theory and applications. Proc Sympos Appl Math AMS. 40 171-179 (1990)

14. Rao, CR, Mitra, SK: Generlized Inverse of matrices and its Application. Wiley, New York (1971)

15. Tian, Y: Completing block matrices with maximal and minimal ranks. Linear Algebra Appl. 321, 327-345 (2000). doi:10.1016/S0024-3795(00)00224-X

16. Tian, Y: The minimal rank of a $3 \times 3$ partial block matrix. Linear and multilinear Algebra. 50, 125-131 (2002). doi:10.1080/03081080290019531

17. Cohen, N, Dancis, J: Maximal rank Hermitian completions of partially specified Hermitian matrices. Linear Algebra Appl. 244, 265-276 (1996)

18. Liu, $Y$, Tian, $Y$ : More on extremal ranks of the matrix expressions $A-B X \pm X^{*} B^{*}$ with statistical applications. Numer Linear Algebra Appl. 15, 307-325 (2008). doi:10.1002/nla.553

19. Liu, $Y$, Tian, Y: Extremal ranks of submatrices in an Hermitian solution to the matrix equation $A X A^{*}=B$ with applications. J Appl Math Comput. 32, 289-301 (2010). doi:10.1007/s12190-009-0251-8

20. Tian, Y, Liu, Y: Extremal ranks of some symmetric matrix expressions with applications. SIAM J Matrix Anal Appl. 28, 890-905 (2006). doi:10.1137/S0895479802415545

21. Mitra, SK: A pair of simulations linear matrix equations $A_{1} X_{1} B_{1}=C_{1}$ and $A_{2} X_{2} B_{2}=C_{2}$ and a programming problem Linear Algebra Appl. 131, 107-123 (1990)

22. Puntanen, S, Styan, GHP, Tian, Y: Three rank formulas associated with the covariance matrices of the BLUE and the OLSE in the general linear model. Econometric Theory. 21, 659-664 (2005)

23. Rao, CR: Unified theory of linear estimation. Sankhyā Ser A. 33, 371-394 (1971)

24. Rao, CR: Representations of best linear unbiased estimators in the Gauss-Markoff model with a singular dispersion matrix. J Multivariate Anal. 3, 276-292 (1973). doi:10.1016/0047-259x(73)90042-0

25. Tian, Y: Upper and lower bounds for ranks of matrix expressions using generalized inverses. Linear Algebra Appl. 355, 187-214 (2002). doi:10.1016/S0024-3795(02)00345-2

26. Tian, Y: Using rank formulas to characterize equalities for Moore-Penrose inverse of matrix products. Appl Math Comput. 147, 581-600 (2004). doi:10.1016/S0096-3003(02)00796-8

27. Tian, Y: More on maximal and minimal ranks of Schur complements with applications. Appl Math Comput. 152, 675-692 (2004). doi:10.1016/S0096-3003(03)00585-X

28. Tian, Y, Cheng, S: The maximal and minimal ranks of A - BXC with applications. New York J Math. 9, 345-362 (2003)

doi:10.1186/1029-242X-2012-54

Cite this article as: Xiong et al:: The maximal and minimal ranks of matrix expression with applications. Journal of Inequalities and Applications 2012 2012:54. 